\title{
NLO Simulations of Chargino Production at the ILC
}

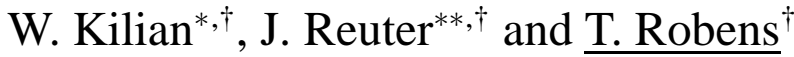 \\ ${ }^{*}$ Fachbereich Physik, University of Siegen, D-57068 Siegen, Germany \\ ${ }^{\dagger}$ Deutsches Elektronen-Synchrotron DESY, D-22603 Hamburg, Germany \\ ${ }^{* *}$ Carleton University, Dep. of Physics, 1125 Colonel By Dr., Ottawa, ON, K1S 5B6, Canada
}

\begin{abstract}
We present an extension of the Monte Carlo Event Generator WH I ZARDwhich includes chargino production at the ILC at NLO. We present two ways of adding photonic contributions. We present results for cross sections and event generation.
\end{abstract}

Keywords: Supersymmetry, NLO, Monte Carlo Event Generator

PACS: 12.15.Lk, 13.40.Ks, 13.66.Hk, 14.80.Ly

\section{INTRODUCTION}

In many GUT models, the masses of charginos tend to be near the lower edge of the superpartner spectrum, and they can be pair-produced at a first-phase ILC with c.m. energy of $500 \mathrm{GeV}$. The precise measurement of their parameters (masses, mixings, and couplings) is a key for uncovering the fundamental properties of the MSSM [1]. Regarding the experimental precision at the ILC, off-shell kinematics for the signal process, and the reducible and irreducible backgrounds [2] need to be included as well as NLO corrections for chargino production at the ILC which are in the percent regime. We here present the inclusion of the latter.

\section{CHARGINO PRODUCTION AT LO AND NLO}

The total fixed-order NLO cross section is given by

$$
\sigma_{\mathrm{tot}}\left(s, m_{e}^{2}\right)=\sigma_{\mathrm{Born}}(s)+\sigma_{\mathrm{v}+\mathrm{s}}\left(s, \Delta E_{\gamma}, m_{e}^{2}\right)+\sigma_{2 \rightarrow 3}\left(s, \Delta E_{\gamma}, m_{e}^{2}\right)
$$

where $s$ is the $\mathrm{cm}$ energy, $m_{e}$ the electron mass, and $\Delta E_{\gamma}$ the soft photon energy cut dividing the photon phase space. The 'virtual' contribution is the interference of the one-loop corrections [3] with the Born term. The collinear and infrared singularities are regulated by $m_{e}$ and $\lambda$, respectively. The dependence on $\lambda$ is eliminated by adding the soft real photon contribution $\sigma_{\text {soft }}=f_{\text {soft }} \sigma_{\text {Born }}(s)$ with a universal soft factor $f_{\text {soft }}\left(\frac{\Delta E_{\gamma}}{\lambda}\right)$ [4]. We break the 'hard' contribution $\sigma_{2 \rightarrow 3}\left(s, \Delta E_{\gamma}, m_{e}^{2}\right)$, i.e., the realradiation process $e^{-} e^{+} \rightarrow \tilde{\chi}_{i}^{-} \tilde{\chi}_{j}^{+} \gamma$, into a collinear and a non-collinear part, separated at a photon acollinearity angle $\Delta \theta_{\gamma}$ relative to the incoming electron or positron: $\sigma_{2 \rightarrow 3}\left(s, \Delta E_{\gamma}, m_{e}^{2}\right)=\sigma_{\text {hard,non-coll }}\left(s, \Delta E_{\gamma}, \Delta \theta_{\gamma}\right)+\sigma_{\text {hard,coll }}\left(s, \Delta E_{\gamma}, \Delta \theta_{\gamma}, m_{e}^{2}\right)$. The collinear 

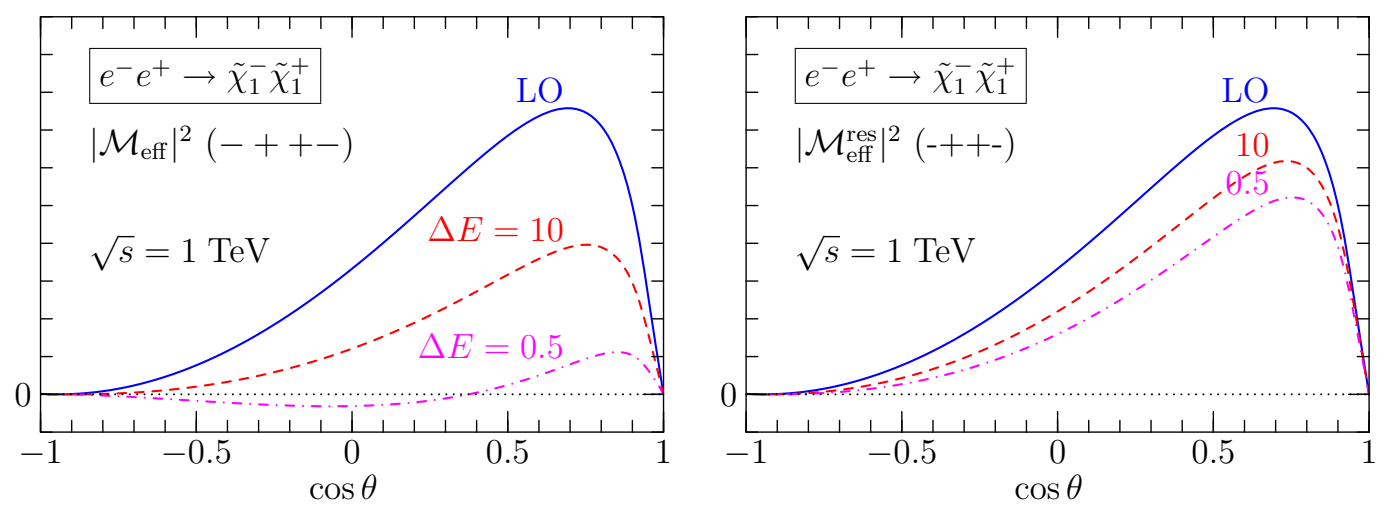

FIGURE 1. $\theta$-dependence of effective squared matrix element $(\sqrt{s}=1 \mathrm{TeV})$.Left figure: fixed order effective matrix element; right figure: effective matrix element with the one-photon ISR part subtracted. Solid line: Born term; dashed: including virtual and soft contributions for $\Delta E_{\gamma}=10 \mathrm{GeV}$; dotted: same with $\Delta E_{\gamma}=0.5 \mathrm{GeV} . \Delta \theta_{\gamma}=1^{\circ}$.

part is approximated by convoluting the Born cross section with a structure function $f\left(x ; \Delta \theta_{\gamma}, \frac{m_{e}^{2}}{s}\right)[5]$. The non-collinear part is generated explicitely.

The total fixed order cross section is implemented in the multi-purpose event generator $\mathrm{O}^{\prime}$ Mega/WHIZARD [6, 7] using a 'user-defined' structure function and an effective matrix element $\left|\mathscr{M}_{\text {eff }}\right|^{2}$ which contains the Born part, the soft-photon factor and the Born-1 loop interference term. In the soft-photon region this approach runs into the problem of negative event weights [8, 9]: for some values of $\theta$, the $2 \rightarrow 2$ part of the NLO-corrected squared matrix element is positive definite by itself only if $\Delta E_{\gamma}$ is sufficiently large and becomes negative otherwise, cf Fig. 1. To still obtain unweighted event samples, an ad-hoc approach is to simply drop events with negative events before proceeding further.

Alternatively, negative event weights can be eliminated using an exponentiated structure function $f_{\text {ISR }}$ which resums higher-order initial radiation [10, 11]. The cancellation of infrared singularities between virtual and real corrections is built-in, so that the main source of negative event weights is eliminated. To combine the ISR-resummed LO result with the additional NLO contributions [3], we subtract from the effective squared matrix element, for each incoming particle, the contribution of one soft photon that has already been accounted for in $\sigma_{\mathrm{s}}$. This defines $\left|\widetilde{\mathscr{M}}_{\text {eff }}\right|^{2}=\left|\mathscr{M}_{\text {eff }}\right|^{2}-2 f_{\text {soft,ISR }}\left|\mathscr{M}_{\text {Born }}\right|^{2}$, which contains the Born term, the virtual and soft collinear contribution with the leadinglogarithmic part of virtual photons and soft collinear emission removed, and soft noncollinear radiation of one photon. Convoluting this with the resummed ISR structure function, we obtain a modified $2 \rightarrow 2$ part of the total cross section which also includes soft and collinear photonic corrections to the Born/one-loop interference. The complete result also contains the hard non-collinear $2 \rightarrow 3$ part. The resummation approach eliminates the problem of negative weights (cf. Fig. 1) such that unweighting of generated events and realistic simulation at NLO are now possible in all regions of phase-space. A final improvement is to also convolute the $2 \rightarrow 3$ part with the ISR structure function [12]. 


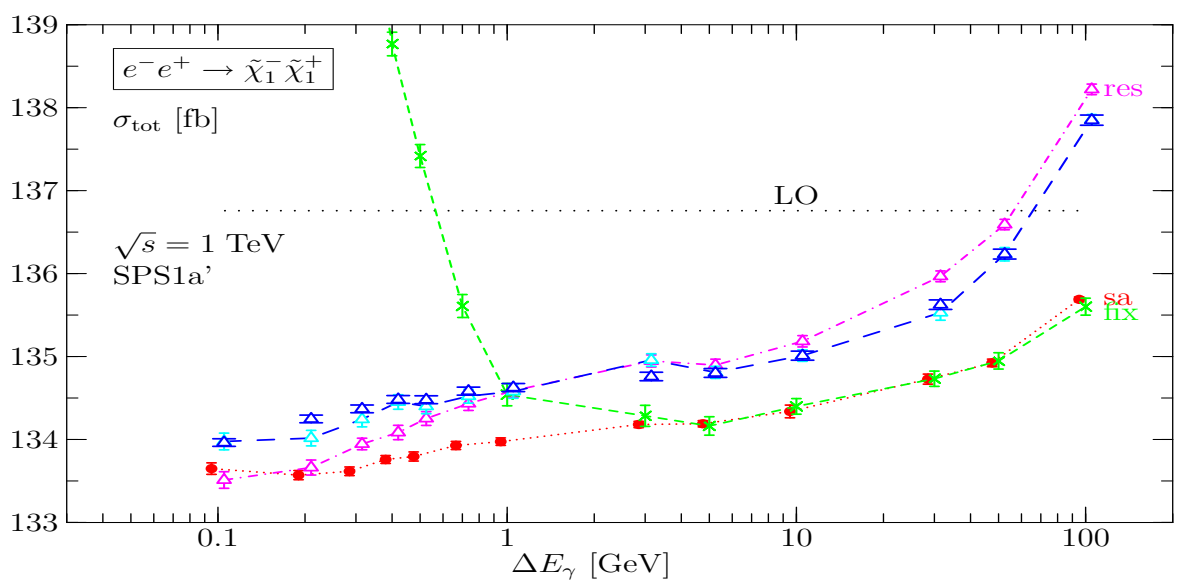

FIGURE 2. Total cross section dependence on $\Delta E_{\gamma}$ : 'sa' (dotted) = fixed-order semianalytic result; 'fix' (dashed) = fixed-order Monte-Carlo result; 'res' (long-dashed) = ISR-resummed Monte-Carlo result; $($ dash-dotted $)=$ same but resummation applied only to the $2 \rightarrow 2$ part. $\Delta \theta_{\gamma}=1^{\circ}$. LO: Born cross section.

\section{RESULTS}

First, we have to check the dependence of the total cross section on the cutoffs $\Delta E_{\gamma}, \Delta \theta_{\gamma}$. Fig. 2 compares the $\Delta E_{\gamma}$ dependence of the numerical results from the semianalytic fixed-order calculation with the Monte-Carlo integration in the fixed-order and in the resummation schemes. The fixed-order Monte-Carlo result agrees with the semianalytic result as long as the cutoff is greater than a few $\mathrm{GeV}$ but departs from it for smaller cutoff values because here, in some parts of phase space, $\left|\mathscr{M}_{\text {eff }}\right|^{2}<0$ is set to zero. The semianalytic fixed-order result is not exactly cutoff-independent, but exhibits a slight rise of the calculated cross section with increasing cutoff (breakdown of the soft approximation). For $\Delta E_{\gamma}=1 \mathrm{GeV}(10 \mathrm{GeV})$ the shift is about $2 \%(5 \%)$ of the total cross section. The fully resummed result shows an increase of about $5 \%$ of the total cross section with respect to the fixed-order result which stays roughly constant until $\Delta E_{\gamma}>10 \mathrm{GeV}$. This is due to higher-order photon radiation.

For the dependence on the collinear cutoff $\Delta \theta_{\gamma}$, the main higher-order effect is associated with photon emission angles below $0.1^{\circ}$. For $\theta_{\gamma}>10^{\circ}$, the collinear approximation breaks down.

In Fig. 3] we show the binned distribution of the chargino production angle as obtained from a sample of unweighted events. It demonstrates that NLO corrections (which, for total cross sections, are in the percent regime and can reach 20\% at the threshold) are important and cannot be accounted for by a constant K factor. In summary, to carefully choose the resummation method and cutoffs will be critical for a truly precise analysis of real ILC data. For more details, cf. [12, 13].

\section{CONCLUSIONS}

We have implemented NLO corrections into the event generator WH I ZARDfor chargino pair-production at the ILC with several approaches for the inclusion of photon radiation. 

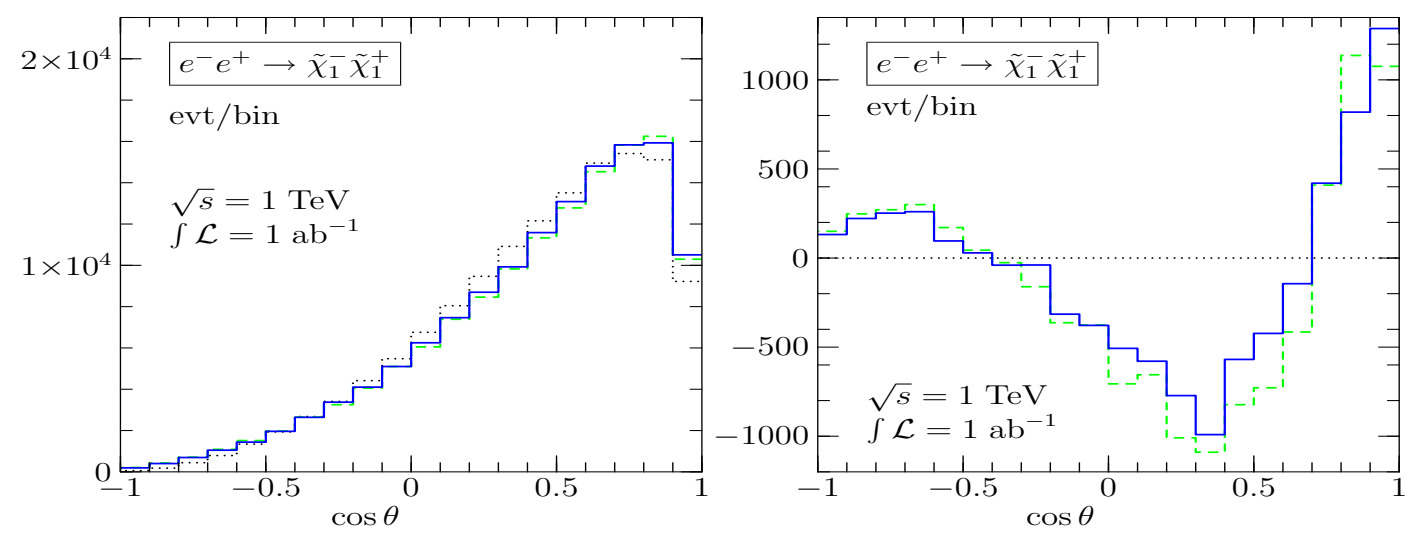

FIGURE 3. Polar scattering angle distribution for an integrated luminosity of $1 \mathrm{ab}^{-1}$ at $\sqrt{s}=1 \mathrm{TeV}$. Left: total number of events per bin; right: difference w.r.t. the Born distribution. LO (dotted) $=$ Born cross section without ISR; fix (dashed) = fixed-order approach; res (full) = resummation approach. Cutoffs: $\Delta E_{\gamma}=3 \mathrm{GeV}$ and $\Delta \theta_{\gamma}=1^{\circ}$.

A careful analysis of the dependence on the cuts $\Delta E_{\gamma}, \Delta \theta$ reveals uncertainties related to higher-order radiation and breakdown of the soft or collinear approximations. The version of the program resumming photons allows to get rid of negative event weights in the simulation, accounts for all yet known higher-order effects, allows for cutoffs small enough that soft- and collinear-approximation artefacts are negligible, and explicitly generates photons where they can be resolved experimentally. Corrections for the decays of charginos and non-factorizing corrections are in the line of future work.

\section{ACKNOWLEDGMENTS}

This work was supported by the German Helmholtz Association, Grant VH-NG-005.

\section{REFERENCES}

1. J. A. Aguilar-Saavedra, et al., Eur. Phys. J. C46, 43-60 (2006), hep-ph/0511344

2. K. Hagiwara et al., Phys. Rev. D73, 055005 (2006), hep-ph/ 0512260

3. T. Fritzsche, and W. Hollik, Nucl. Phys. Proc. Suppl. 135, 102-106 (2004), hep-ph/0 407095

4. A. Denner, Fortschr. Phys. 41, 307-420 (1993).

5. M. Bohm, and S. Dittmaier, Nucl. Phys. B409, 3-21 (1993).

6. M. Moretti, T. Ohl, and J. Reuter, O’Mega (2001), hep-ph/0102195

7. W. Kilian, Whizard 1.0 (2001), LC-TOOL-2001-039.

8. M. Bohm, A. Denner, and W. Hollik, Nucl. Phys. B304, 687 (1988).

9. R. Kleiss, et al., "MONTE CARLOS FOR ELECTROWEAK PHYSICS," in Proceedings, Z physics at LEP 1, vol. 3, CERN, Geneva, 1989.

10. V. N. Gribov, and L. N. Lipatov, Sov. J. Nucl. Phys. 15, 675-684 (1972).

11. M. Skrzypek, and S. Jadach, Z. Phys. C49, 577-584 (1991).

12. W. Kilian, J. Reuter, and T. Robens, accepted in Eur. Phys. J. C (2006), hep-ph / 0607127.

13. T. Robens, Event Generation for Next to Leading Order Chargino Production at the International Linear Collider, Ph.D. thesis (2006), hep-ph/0610401. 